\title{
Theoretical Investigation of X-Ray Absorption near Edge Spectroscopy (XANES) Angular Dependence of Aligned Carbon Nanotubes Grown by DC HF CVD Process
}

\author{
Bridinette Thiodjio Sendja ${ }^{*}$, Rolant Eba Medjo², Jeannot Mane Mane1, \\ Germain Hubert Ben-Bolie ${ }^{3}$, Pierre Owono Ateba ${ }^{3}$ \\ ${ }^{1}$ Ecole Nationale Supérieure Polytechnique (National Advanced School of Engineering), Department of \\ Mathematics and Physical Sciences, University of Yaoundé I, Yaounde, Cameroon \\ ${ }^{2}$ Physics Department, Faculty of Science, University of Douala, Douala, Cameroon \\ ${ }^{3}$ Physics Department, Faculty of Science, University of Yaoundé I, Yaounde, Cameroon \\ Email: sbridine@yahoo.fr
}

Received 6 March 2015; accepted 5 May 2015; published 12 May 2015

Copyright (C 2015 by authors and Scientific Research Publishing Inc.

This work is licensed under the Creative Commons Attribution International License (CC BY).

http://creativecommons.org/licenses/by/4.0/

c) (i) Open Access

\begin{abstract}
Carbon nanotubes (CNTs) grown on plain substrates $\mathrm{SiO}_{2} / \mathrm{Si}(100)$ by a direct current and hot filaments catalytic chemical vapor deposition process have been studied by synchrotron X-ray absorption near edge spectroscopy (XANES) technique to theoretically investigate the angular-dependence of carbone (C) K-edge $\pi^{*}$ and $\sigma^{*}$ transitions. Experimental XANES spectra show that $\pi^{*}$ resonance increases with the incidence angle from normal to grazing incidence angle while $\sigma^{*}$ resonance decreases. This has been explained by the sine-square and cosine-square dependencies of $\pi^{*}$ and $\sigma^{*}$ intensities, respectively. These results were confirmed by theoretical XANES curves of highly oriented pyrolytic graphite (HOPG) and CNTs plotted versus incidence angle. It has been shown that $\pi^{*}$ and $\sigma^{*}$ transitions strongly depend on the nature of polarized light (linearly or circularly). At the linear polarized light, $\pi^{*}$ resonance is a preference as well as at right-circular polarized. At the left-circular polarized light, $\sigma^{*}$ resonance is a preference. The $\pi^{*}$ intensities are high at parallel orientation and the $\sigma^{*}$ intensities are low at normal orientation. The smallest $\pi^{*}$ intensity is noticed at normal orientation, where the $\pi^{*}$ orbitals are supposed to be lying parallel to the surface plane for perfectly aligned HOPG or CNTs. This explains the incomplete extinction of $\pi^{*}$ intensity. We noticed at parallel orientation a region where any $\pi^{*}$ and $\sigma^{*}$ transitions did not expect because of the lack of polarization light.
\end{abstract}

${ }^{*}$ Corresponding author.

How to cite this paper: Sendja, B.T., Medjo, R.E., Mane, J.M., Ben-Bolie, G.H. and Ateba, P.O. (2015) Theoretical Investigation of X-Ray Absorption near Edge Spectroscopy (XANES) Angular Dependence of Aligned Carbon Nanotubes Grown by DC HF CVD Process. Materials Sciences and Applications, 6, 373-390. http://dx.doi.org/10.4236/msa.2015.65043 
Keywords

Angular Dependence, XANES, Normal Incidence, Grazing Incidence, Carbon Nanotubes, Intensity

\section{Introduction}

Since the discovery of carbon nanotubes in 1991 by Iijima [1], a considerable effort is currently devoted particularly to their field emission property. This property as well as many others such as a high aspect ratio (ratio of the length to the external diameter), a reasonable work function, a chemical inertness and a mechanical robustness provides them related applications and makes them very interesting on a technological point of view. Preparation of such samples implies the growth of bundle or individual aligned nanotubes standing on flat substrate. Catalytic Chemical Vapor Deposition (CCVD) techniques using plasma-enhancement (PE-CCVD) and direct current and hot filaments (DC HF CCVD) are the best adapted to fulfill these requirements as the local electric field orientates the growth normal to the surface. Thus a fine and localized control of the nucleation and growth of oriented nanotubes can be achieved from catalyst made up of transition metal particles (Fe, Co, Ni) spread onto the surface [2].

To more understand the properties and applications of carbon nanotubes, more techniques have been used such as Transmission Electron Microscopy (TEM) and Scanning Electron Microscopy (SEM) techniques which provide the qualitative overview study of alignment of carbon nanotubes [3]-[5]. Grazing Incidence Small Angle X-ray Scattering (GISAXS) by contrast allows the quantitative study of their alignment, their structure and their morphology [6] and their angular dependence [7]. X-ray Absorption Spectroscopy (XAS) as more techniques has its advantage to probe the spatial orientation of sample by varying the direction of light polarization and the electronic density of unoccupied density of state of a system by varying the photon energy around an absorption edge of an atom. Recently, much progress has been done to understand the spatial orientation and electronic structure of aligned carbon nanotubes using X-ray Absorption Near Edge Spectroscopy (XANES) technique [8]-[10]. No more attention is paid to the study of angular dependence of aligned carbon nanotubes instead of the unlimited researches made on carbon nanotubes. These last years, angle-resolved has been investigated [11][14] showing the sine-squared and cosine-squared dependencies of $\pi^{*}$ and $\sigma^{*}$ intensities, respectively. Chiou et al. found enhanced $\pi^{*}$ and $\sigma^{*}$ intensities at the tips of multiwalled carbon nanotube (MWNT) arrays [11]. More recently, angular dependence of aligned carbon nanotubes has been studied by GISAXS technique to quantify the disorder degree of their orientation since they can be affected by some other species using during the preparation sample [7].

In this paper, we theoretically investigate the angular dependence of aligned carbon nanotubes using XANES technique in order to deep the orientation and the final state of density of state (DOS) around the free-interlayerelectron region for the study of their anisotropy. We used highly pyrolytic graphique (HOPG) and carbon nanotubes (CNTs) XANES spectra to investigate the angular dependence of the carbon nanotubes studied by Grazing Incidence Small Angle X-ray Scattering (GISAXS) on previous paper [7] and introduced with X-ray Absorption Spectroscopy by Mane et al. [15]. The experimental results are used for simulation to approach the XANES angular dependence theory to the experimental conditions. The aim is to study theoretically the behavior of $\pi^{*}$ and $\sigma^{*}$ intensities angular dependence at grazing and normal incidence and compare with the tendency found in the literature.

\section{XANES Experiments and Theoretical Analysis of Angular Dependence}

The different steps of substrate preparation and CNTs growth could be found in references [5] [10] [15].

\subsection{XANES Experiments Process}

Substrate preparation and CNTs growth conditions have been developed elsewhere [7] [10] [15]. Our attention will be focus only on XANES experiment. The carbon K-edge XANES measurements were carried out on the SACEMOR beamline at the Laboratoire pour l'Utilisation du Rayonnement Electromagnétique (LURE, ORSAY, France) on the VUV Super-Aco storage ring. The tutorial grating monochromator (TGM) was used with 1200 
lines $\mathrm{Nm}^{-1}$ grating (better resolution than 0.2 at $\mathrm{C}$ K-edge). The spectra were recorded in total-electron-yield detection (TEY) and partial electron yield (PEY), which is expected to be less surface sensitive. Then, were normalized to the copper grid spectrum freshly coated with gold. The XANES data were collected at characteristics angles such as grazing incidence (with $\theta=90^{\circ}$, $\mathrm{E}$ is normal to the surface) and normal incidence (with $\theta=$ $0^{\circ}$, E parallel to the surface), where $\theta$ denotes the angle between the sample normal and the direction of the electric vector (E) of the X-ray beam (see Figure 1). Energies were calibrated with $0.1 \mathrm{eV}$ step to the carbon ionization potential (IP) and the error on the N K-edge is expected to be more than $0.4 \mathrm{eV}$. More details could be found in Ref. [10]. In order to extract quantitative information, two nitrogen-containing polymer references poly acrylonitrile (PAN) were used as a reference for the terminal nitrile configuration, and (P2VC) as a reference for nitrogen in pyrolytic configuration where the isolated pair of electrons is out of the aromatic plane.

Line shape analyses of the individual C1s lines were carried out using home-made software. The data were first smoothed and subsequently deconvoluted, including both Gaussian broadening (accounting for experimental resolution, electron-phonon interaction and amorphization effects) and Lorentzian broadening (accounting for the life time of the core hole fixed at $0.21 \mathrm{eV}$ for the $\mathrm{C} 1 \mathrm{~s}$ lines). Threshold functions of the $\pi \rightarrow \pi^{*}$ and $\sigma \rightarrow \sigma^{*}$ transitions, which were Heaviside step functions broadened by the Gaussian experimental resolution, were added to these individual contributions. The asymmetry parameter was fixed at 0.1 for $\pi^{*}$ resonance and at 0.07 for $\sigma^{*}$ resonance. Thus, the base line $\pi^{*}$ transition was 0.14 for graphite at grazing and normal incidence and was 0.15 for carbon nanotubes $\pi^{*}$ and $\sigma^{*}$ transition. While, the base line of $\sigma^{*}$ transition was fixed at 0.16 for graphite at grazing and normal incidence.

\subsection{Theoretical Analysis of XANES Angular Dependence}

Knowing that, Pauli's exclusion principle requires that the final quantum state was unoccupied before absorption took place. Quantum mechanics shows us that the probability of an optical transition by the absorption of a photon from an initial state $i$ to final state $f$ is proportional to the square of the transition dipole matrix element:

$$
P_{\text {if }} \propto|\langle f|E \cdot r| i\rangle|^{2}
$$

When the energy of photon is equal to the difference of energy between two states involved in an allowed electronic transition, the photon is resonant with that transition. The transition probably assimilated to the corresponding intensity could be expressed as, according to Fermi's gold rule [16]:

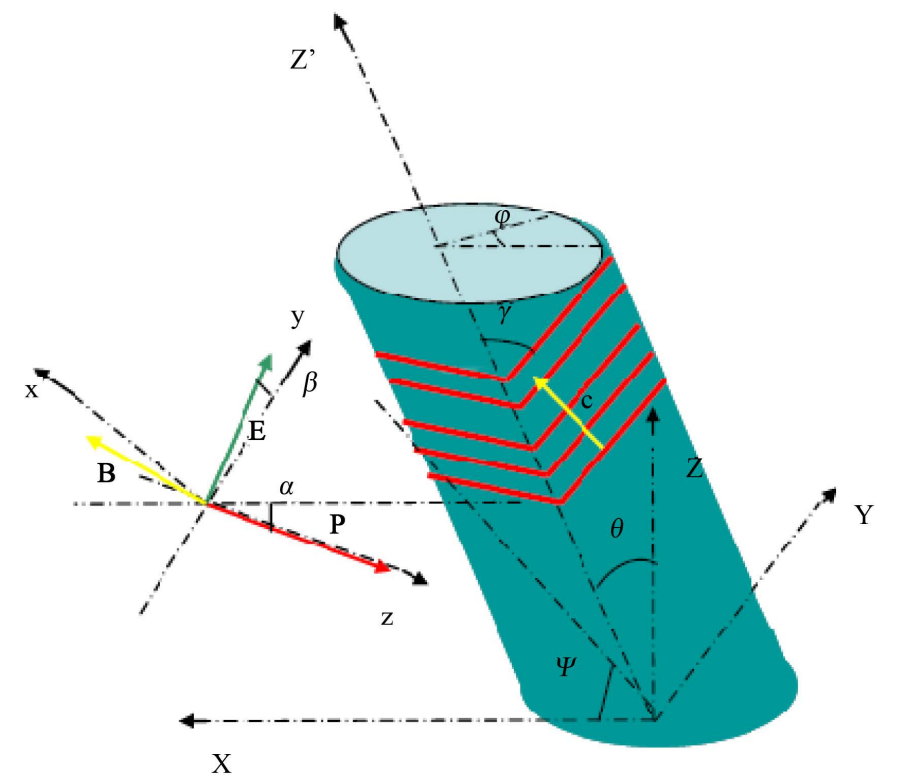

Figure 1. Incident geometry of $\pi^{*}$ and $\sigma^{*}$ orbitals for a vertical CNT tube axis along $z$ with respect to the incident beam and the electric field vector $E$ which remains perpendicular to the incident X-ray beam [15]. 


$$
I=\left(\frac{1}{h}\right)|\langle f|E \cdot r| i\rangle|^{2} P_{f}(E)
$$

$E$ is the electric field vector, $r$ is the position vector and $h$ is a Plank constant. $f$ and $i$ are the final and initial wave state, respectively and $P_{f}(E)$ is the density of final states. To quantitatively understand the results of XANES data analysis, we have to evaluate the angular dependencies that would be expected for two main possible orientations of carbon nanotubes [17].

Assuming the more general case of carbon nanofibers where the graphitic basal planes are oriented with a conical polar angle $\gamma$ relative to the fiber axis $A A^{\prime}$ [15]. In the case of the carbon nanotube, $\gamma$ is equal to zero. The XAS signal is integrated over all the $\pi$ orbitals along the $\varphi$ azimuthal angle around the axis of the carbon nanostructure (Figure 1). Let us consider the case where the carbon nanotubes are grown on a flat substrate with a random polar angle $\theta$ relative to the normal axis $Z Z^{\prime}$ to the substrate. In that case, a random orientation normal to the surface is equivalent to $\theta=0$ and the random orientation parallel to the substrate is equivalent to $\theta=\pi / 2$.

Knowing that the light impinges the surface at an incidence angle $\alpha$ relative to the $(X, Y)$ plane of the substrate with a Poynting polarization vector $P$ in the $z$ direction and the electric vector $E$ is within the plan $(x, y, 0)$ with an angle $\beta$ relative to an horizontal orientation of the electric field in the propagating light (Figure 1), which is generally encountered in synchrotron light. We can consider two particular points with $\beta=0^{\circ}$ where the light is linearly polarized and $\beta=45^{\circ}$ where the light is circularly polarized.

Assuming a general elliptic polarization of the light $0^{\circ} \leq \beta \leq 45^{\circ}$ the intensity of the absorption with a transition to $\pi^{*}$ states of the carbon nanostructures can be expressed by integrating over all azimuthal angles $\varphi[15]$ :

$$
\begin{aligned}
I_{\pi}(\alpha, \beta, \gamma, \theta)= & \frac{N I_{\pi}^{0}}{4}\left\{\cos ^{2} \theta\left[\left(1-3 \sin ^{2} \gamma\right)\left(1-3 \cos ^{2} \beta \cos ^{2} \alpha\right)\right]\right. \\
& \left.+\left(1+\cos ^{2} \beta \cos ^{2} \alpha\right)+\sin ^{2} \gamma\left(1-3 \cos ^{2} \beta \cos ^{2} \alpha\right)\right\}
\end{aligned}
$$

where $N$ is the atomic density of carbon atoms and $I_{\pi}^{0}$ is a maximum experimental value of $\pi^{*}$ intensity. To determine the intensity of $\sigma^{*}$ state, which has $3 \sigma$ orbitals for each $\pi$ orbital, we multiply the $\pi^{*}$ state intensity by 3 and replace $\gamma$ by $\gamma+\pi / 2$, and we can then obtain:

$$
I_{\sigma}(\alpha, \beta, \gamma, \theta)=\frac{3}{2}\left[1-I_{\pi}(\alpha, \beta, \gamma, \theta)\right] .
$$

Let us continue by examining the cases of linearly and circularly polarized light. The case of linear polarized light, the angle $\beta=0^{\circ}$, thus the $\pi^{*}$ state intensity can be expressed as follow:

$$
I_{\pi}(\alpha, 0, \gamma, \theta)=\frac{N I_{\pi}^{0}}{4}\left\{\cos ^{2} \theta\left[\left(1-3 \sin ^{2} \gamma\right)\left(1-3 \cos ^{2} \alpha\right)\right]+\left(1+\cos ^{2} \alpha\right)+\sin ^{2} \gamma\left(1-3 \cos ^{2} \alpha\right)\right\} .
$$

And the $\sigma^{*}$ state intensity as:

$$
I_{\sigma}(\alpha, 0, \gamma, \theta)=\frac{3}{2}\left[1-I_{\pi}(\alpha, 0, \gamma, \theta)\right] .
$$

While the case of circular polarized light, the angle $\beta=\pi / 4$, thus the $\pi^{*}$ state intensity can be expressed as follow:

$$
I_{\pi}\left(\alpha, \frac{\pi}{4}, \gamma, \theta\right)=\frac{N I_{\pi}^{0}}{4}\left\{\cos ^{2} \theta\left[\left(1-3 \sin ^{2} \gamma\right)\left(1-\frac{3}{2} \cos ^{2} \alpha\right)\right]+\left(1+\frac{1}{2} \cos ^{2} \alpha\right)+\sin ^{2} \gamma\left(1-\frac{3}{2} \cos ^{2} \alpha\right)\right\} .
$$

And the $\sigma^{*}$ state intensity as:

$$
I_{\sigma}\left(\alpha, \frac{\pi}{4}, \gamma, \theta\right)=\frac{3}{2}\left[1-I_{\pi}\left(\alpha, \frac{\pi}{4}, \gamma, \theta\right)\right] .
$$

We focus our investigation on the HOPG and the CNTs in order to fully understand the angular dependence of their $\pi^{*}$ and $\sigma^{*}$ transitions.

For HOPG graphite, the value of $\gamma$ is equal to $\frac{\pi}{2}$, so, we can develop the $\pi^{*}$ state intensity Equation (6) 
staying in the case of linear polarized light and get the following equation:

$$
I_{\pi}\left(\alpha, 0, \frac{\pi}{2}, \theta\right)=\frac{N I_{\pi}^{0}}{2}\left[-\cos ^{2} \theta\left(1-3 \cos ^{2} \alpha\right)+\left(1-\cos ^{2} \alpha\right)\right] .
$$

And the $\sigma^{*}$ state intensity can be expressed as:

$$
I_{\sigma}\left(\alpha, 0, \frac{\pi}{2}, \theta\right)=\frac{3}{2}\left[1-I_{\pi}\left(\alpha, 0, \frac{\pi}{2}, \theta\right)\right] .
$$

In the same way, we can get the following formula of $\pi^{*}$ and $\sigma^{*}$ state intensities considering the circular polarized light. Thus:

$$
I_{\pi}\left(\alpha, \frac{\pi}{4}, \frac{\pi}{2}, \theta\right)=\frac{N I_{\pi}^{0}}{4}\left[-2 \cos ^{2} \theta\left(1-\frac{3}{2} \cos ^{2} \alpha\right)+\left(2-\cos ^{2} \alpha\right)\right] .
$$

And the intensity of $\sigma^{*}$ state is:

$$
I_{\sigma}\left(\alpha, \frac{\pi}{4}, \frac{\pi}{2}, \theta\right)=\frac{3}{2}\left[1-I_{\pi}\left(\alpha, \frac{\pi}{4}, \frac{\pi}{2}, \theta\right)\right] .
$$

Now, taking the case of carbon nanotubes where the $\gamma$ is equal to zero, we obtain for the linear polarized light:

$$
I_{\pi}(\alpha, 0,0, \theta)=\frac{N I_{\pi}^{0}}{4}\left[\cos ^{2} \theta\left(1-3 \cos ^{2} \alpha\right)+\left(2+\cos ^{2} \alpha\right)\right]
$$

And:

$$
I_{\sigma}(\alpha, 0,0, \theta)=\frac{3}{2}\left[1-I_{\pi}(\alpha, 0,0, \theta)\right]
$$

For the case of circular polarized light, we have the following expressions of $\pi^{*}$ and $\sigma^{*}$ state intensities of carbon nanotubes:

$$
I_{\pi}\left(\alpha, \frac{\pi}{4}, 0, \theta\right)=\frac{N I_{\pi}^{0}}{4}\left[\cos ^{2} \theta\left(1-\frac{3}{2} \cos ^{2} \alpha\right)+\left(1+\frac{1}{2} \cos ^{2} \alpha\right)\right] .
$$

And:

$$
I_{\sigma}\left(\alpha, \frac{\pi}{4}, 0, \theta\right)=\frac{3}{2}\left[1-I_{\pi}\left(\alpha, \frac{\pi}{4}, 0, \theta\right)\right] .
$$

In the previous papers [10] [15], it has been shown that HOPG and CNTs have two main orientations normal (perpendicular) to the surface substrate with the $\theta$ angle equal to zero and parallel to the surface substrate with $\theta$ angle equal to $\frac{\pi}{2}$. Obviously, $\pi^{*}$ and $\sigma^{*}$ state intensities of HOPG and CNTs could be finally rewritten in these conditions.

HOPG in the linear polarized light, we can get for normal orientation $(\theta=0)$ :

$$
I_{\pi}\left(\alpha, 0, \frac{\pi}{2}, 0\right)=N I_{\pi}^{0} \cos ^{2} \theta
$$

And:

$$
I_{\sigma}\left(\alpha, 0, \frac{\pi}{2}, 0\right)=\frac{3}{2}\left[1-I_{\pi}\left(\alpha, 0, \frac{\pi}{2}, 0\right)\right] .
$$

HOPG in the circular polarized light, we obtain for normal orientation $(\theta=0)$ :

$$
I_{\pi}\left(\alpha, \frac{\pi}{4}, \frac{\pi}{2}, 0\right)=\frac{N I_{\pi}^{0}}{2}\left(\cos ^{2} \alpha\right)
$$


And:

$$
I_{\sigma}\left(\alpha, \frac{\pi}{4}, \frac{\pi}{2}, 0\right)=\frac{3}{2}\left[1-I_{\pi}\left(\alpha, \frac{\pi}{4}, \frac{\pi}{2}, 0\right)\right] .
$$

HOPG in the linear polarized light, we can have for parallel orientation $\left(\theta=\frac{\pi}{2}\right)$ :

$$
I_{\pi}\left(\alpha, 0, \frac{\pi}{2}, \frac{\pi}{2}\right)=\frac{N I_{\pi}^{0}}{2}\left(1-\cos ^{2} \theta\right) .
$$

And:

$$
I_{\sigma}\left(\alpha, 0, \frac{\pi}{2}, \frac{\pi}{2}\right)=\frac{3}{2}\left[1-I_{\pi}\left(\alpha, 0, \frac{\pi}{2}, \frac{\pi}{2}\right)\right]
$$

HOPG in the circular polarized light, one can get for parallel orientation $\left(\theta=\frac{\pi}{2}\right)$ :

$$
I_{\pi}\left(\alpha, \frac{\pi}{4}, \frac{\pi}{2}, \frac{\pi}{2}\right)=\frac{N I_{\pi}^{0}}{4}\left(2-\cos ^{2} \alpha\right)
$$

And:

$$
I_{\sigma}\left(\alpha, \frac{\pi}{4}, \frac{\pi}{2}, \frac{\pi}{2}\right)=\frac{3}{2}\left[1-I_{\pi}\left(\alpha, \frac{\pi}{4}, \frac{\pi}{2}, \frac{\pi}{2}\right)\right] .
$$

Let us now taking the case of carbon nanotubes in these two configurations, we have: CNTs in the linear polarized light, with normal orientation $(\theta=0)$ :

$$
I_{\pi}(\alpha, 0,0,0)=\frac{N I_{\pi}^{0}}{2}\left(1-\cos ^{2} \alpha\right) .
$$

And:

$$
I_{\sigma}(\alpha, 0,0,0)=\frac{3}{2}\left[1-I_{\pi}(\alpha, 0,0,0)\right] .
$$

CNTs in the circular polarized light, with normal orientation $(\theta=0)$ :

$$
I_{\pi}\left(\alpha, \frac{\pi}{4}, 0,0\right)=\frac{N I_{\pi}^{0}}{4}\left(3-2 \cos ^{2} \alpha\right) .
$$

And:

$$
I_{\sigma}\left(\alpha, \frac{\pi}{4}, 0,0\right)=\frac{3}{2}\left[1-I_{\pi}\left(\alpha, \frac{\pi}{4}, 0,0\right)\right] .
$$

CNTs in the linear polarized light, with parallel orientation $\left(\theta=\frac{\pi}{2}\right)$ :

$$
I_{\pi}\left(\alpha, 0,0, \frac{\pi}{2}\right)=\frac{N I_{\pi}^{0}}{4}\left(1+\cos ^{2} \alpha\right) .
$$

And:

$$
I_{\sigma}\left(\alpha, 0,0, \frac{\pi}{2}\right)=\frac{3}{2}\left[1-I_{\pi}\left(\alpha, 0,0, \frac{\pi}{2}\right)\right]
$$

CNTs in the circular polarized light, with parallel orientation $\left(\theta=\frac{\pi}{2}\right)$ : 


$$
I_{\pi}\left(\alpha, \frac{\pi}{4}, 0, \frac{\pi}{2}\right)=\frac{N I_{\pi}^{0}}{4}\left(1+\frac{1}{2} \cos ^{2} \alpha\right)
$$

And:

$$
I_{\sigma}\left(\alpha, \frac{\pi}{4}, 0, \frac{\pi}{2}\right)=\frac{3}{2}\left[1-I_{\pi}\left(\alpha, \frac{\pi}{4}, 0, \frac{\pi}{2}\right)\right] .
$$

\section{Results and Discussion}

SEM and TEM analysis of orientation of CNTs grown by HF PE CCVD process have been presented elsewhere [7] [10] [15]. From SEM and TEM images, we had observed highly contrasted Co particles inside the tubes and on top of the tubes. Accurate analysis of SEM and TEM images can give an idea of the main orientation of carbon nanotubes and allow the estimation of their outer diameter and length. However, their analysis is only qualitative and cannot provide good information about the angle dependence. XANES, by contrast, is suitable for the appropriate study of angular dependence of $\pi$ and $\sigma$ resonances obtained after heating X-ray photon on a 1s C K edge core hole of sample. DOS calculations were used to possible assignment of the features of the $\mathrm{C}(\mathrm{K})$ absorption structure to specific states of the Brillouin zone in the theoretical band structure. We focus our attention only on the region between peaks $\mathrm{A}$ and $\mathrm{B}$ assigned to $\pi^{*}$ and $\sigma^{*}$ resonances, respectively. The above region is not our interest of angular dependences of these transitions. Theoretical curves of angular dependence of $\pi^{*}$ and $\sigma^{*}$ resonances have been obtained by simulation taken as input parameters the values extracted from XANES analysis.

\subsection{XANES Analysis of Orientation of HOPG Grown by DC HF CCVD Process}

For a good understanding of polarization trends and angular dependence for Carbon nanotube samples, we first analyze the HOPG XANES spectra that are considered as reference to our analysis. Typical CK-edge HOPG spectra, at normal (normal orientation $\theta=0^{\circ}$ ) and grazing (parallel orientation $\theta=\pi / 2$ ) incidence angles are plotted and shown in Figure 2(a) and Figure 2(b), respectively.

In Figure 2(a) plotted at normal incidence angle (normal orientation), A and B are localized respectively at $285.45 \mathrm{eV}$ and $292.93 \mathrm{eV}$. They are attributed to unoccupied $\pi^{*}$ resonance and $\sigma^{*}$ resonance, respectively [9] [10]. The final-state band and Brillouin zone region of peak $\mathrm{A}$ is associated to $\pi_{0}$ near $\mathrm{Q}$ at $2 \mathrm{eV}$ above the Fermi level while peak B is attributed to $\sigma_{1}, \sigma_{2}$ in the Brillouin zone $\Gamma \rightarrow \mathrm{Q}$. $\mathrm{A}_{1}$ and $\mathrm{A}_{2}$ are seen at $287.21 \mathrm{eV}$ and $288.49 \mathrm{eV}$, respectively. $\mathrm{A}_{1}$ is currently associated to the free-electron-like interlayer states in graphite and corresponds to $\pi^{*}$ resonance [9] [10] [18]. It has been shown that it may due to the adsorption of some molecules such as $\mathrm{CO}, \mathrm{CO}_{2}$ and water [15]. $\mathrm{A}_{2}$ is also attributed to the free-electron-like interlayer states and correspond to $\pi^{*}$ resonance. It could be due to the adsorption of functionalized states $C=C$. Concerning $A_{3}$, found at $291.78 \mathrm{eV}$ and currently observed on some HOPG XAS spectra, it corresponds to $\sigma^{*} \mathrm{C}-\mathrm{O}$ bond resonance. $\mathrm{A}_{1}, \mathrm{~A}_{2}$ and $\mathrm{A}_{3}$ peaks are not far from those found in Ref. [15].

Looking for Figure 2(b) taken at grazing incidence angle (parallel orientation), we can see that the main peaks A attributed to unoccupied $\pi^{*}$ resonance occurs at $285.40 \mathrm{eV}$ and $\mathrm{B}$ attributed to unoccupied $\sigma^{*}$ resonance occurs at $292.70 \mathrm{eV}$ [9] [10]. Their final-state band and Brillouin zone region are $\pi_{0}$ near $\mathrm{Q}$ at $2 \mathrm{eV}$ above the Fermi level and $\sigma_{1}, \sigma_{2}$ in the brilloun zone $\Gamma \rightarrow \mathrm{Q}$, respectively. Between the $\mathrm{A}$ and $\mathrm{B}$ peaks, we found three peaks named $A_{1}, A_{2}$ and $A_{3} . A_{1}$ is the weak one localized near $287.20 \mathrm{eV}$. It was associated to the free-electron-like interlayer states in graphite and corresponds to $\pi^{*}$ resonance. It may due to the adsorption of some molecules such as $\mathrm{CO}, \mathrm{CO}_{2}$ and water. $\mathrm{A}_{2}$ is localized at $288.49 \mathrm{eV}$ and corresponds to $\pi^{*}$ resonance which could be due to the adsorption of functionalized states $\mathrm{C}=\mathrm{C}$. $\mathrm{A}_{3}$ found at $291.78 \mathrm{eV}$ corresponds to $\sigma^{*}$ of $\mathrm{C}-\mathrm{O}$ bond resonance.

While analyzing Figure 2(a) taken at normal incidence, the intensity of A peak corresponding to $\pi^{*}$ resonance found to be equal to $0.43 \mathrm{c} / \mathrm{s}$ and the one of B peak corresponding to $\sigma^{*}$ resonance is equal to $3.80 \mathrm{c} / \mathrm{s}$. Concerning Figure 2(b) taken at grazing incidence angle, the intensity of A peak corresponding to $\pi^{*}$ resonance found to be equal to $1.71 \mathrm{c} / \mathrm{s}$ and the one of B peak corresponding to $\sigma^{*}$ resonance is equal to $2.46 \mathrm{c} / \mathrm{s}$. We define the orientation parameter (OP) which indicates the tendency of the orientation as the ratio of unique $\pi$-type feature intensities over the unique $\sigma$-type feature intensity. The OP parameter calculated from Table 1 of XANES 


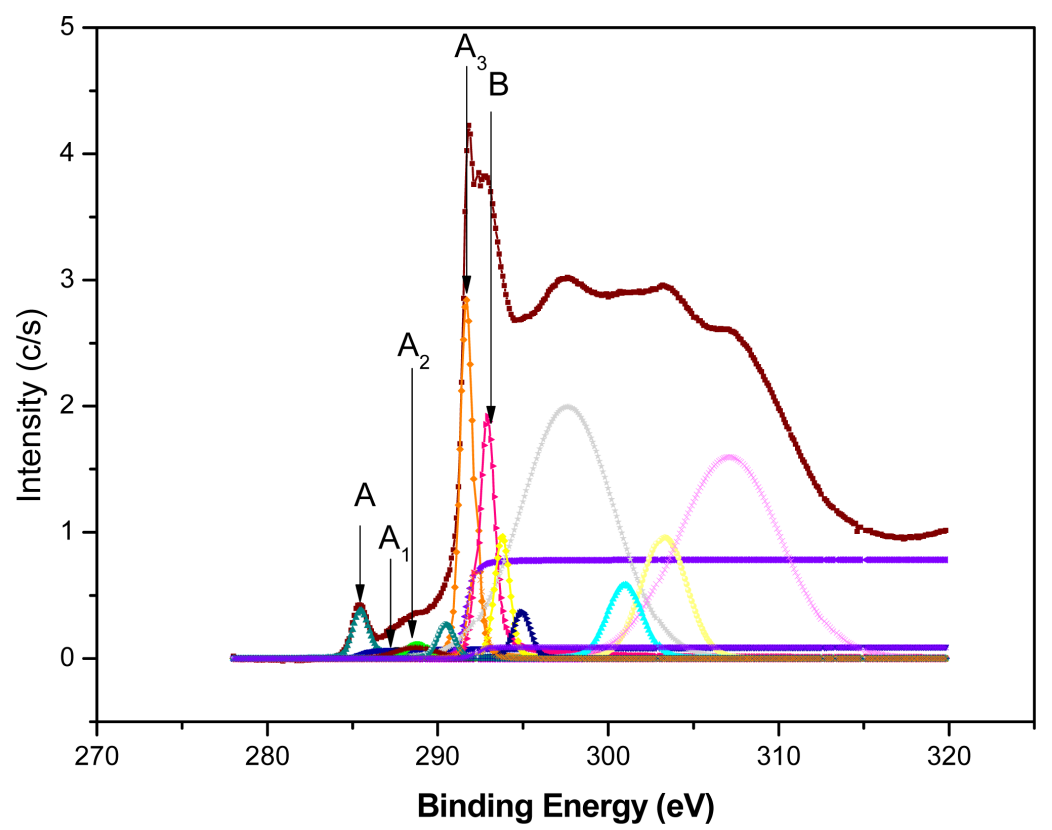

(a)

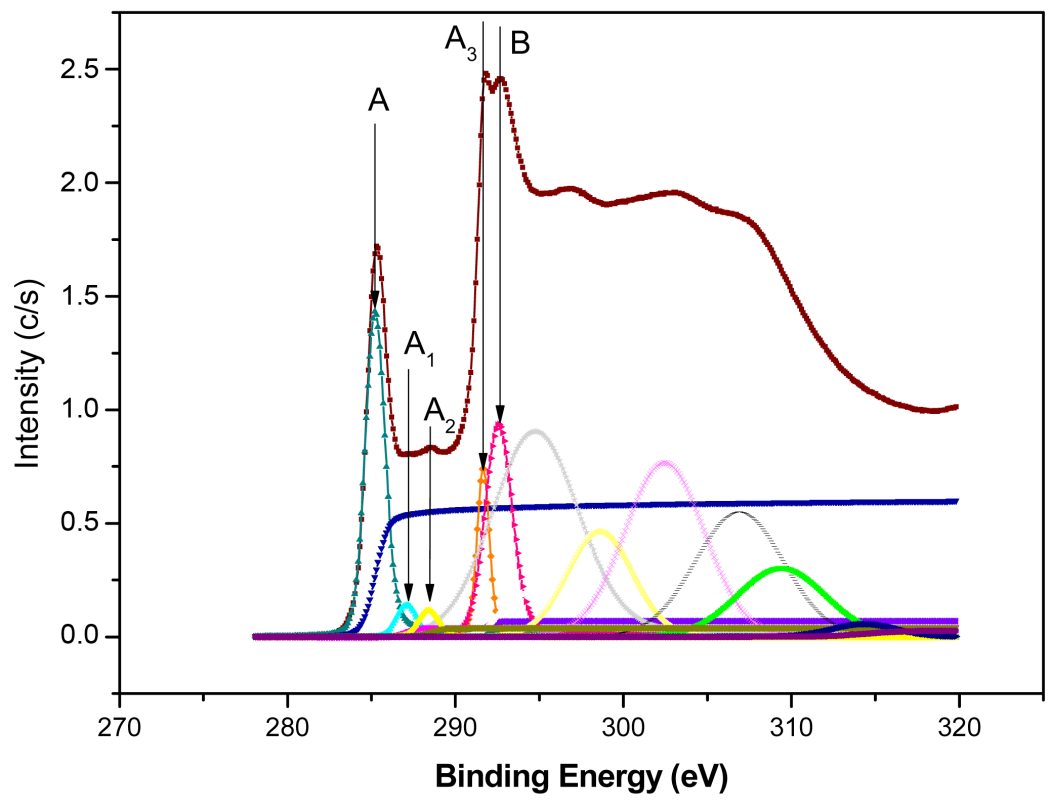

(b)

Figure 2. (a) Graphite experimental and simulated superimposed XANES spectra at normal incidence angle. $\mathrm{A}, \mathrm{A}_{1}, \mathrm{~A}_{2}, \mathrm{~A}_{3}$ and $\mathrm{B}$ are the features assigned to $\pi^{*}$ or $\sigma^{*}$ transitions. Their final state band and Brillouin zone are presented in Table 1 . The asymmetry parameter of $\mathrm{A}$ is equal to 0.10 while the one of $\mathrm{B}$ is equal to 0.07 . The line base value of $A$ is 0.14 while the one of $B$ is 0.16 . The asymmetry and base line parameters of the intermediate peaks $\left(A_{1}, A_{2}\right.$ and $\left.A_{3}\right)$ are equal to zero. (b) Graphite experimental and simulated superimposed XANES spectra at grazing incidence angle. $\mathrm{A}, \mathrm{A}_{1}, \mathrm{~A}_{2}, \mathrm{~A}_{3}$ and $\mathrm{B}$ are the features assigned to $\pi^{*}$ or $\sigma^{*}$ transitions. Their final state band and Brillouin zone are presented in Table 2 . The asymmetry parameter of $\mathrm{A}$ is equal to 0.10 while the one of $\mathrm{B}$ is equal to 0.07 . The line base value of $A$ is 0.14 while the one of $B$ is 0.16 . The asymmetry and base line parameters of the intermediate peaks $\left(A_{1}, A_{2}\right.$ and $\left.A_{3}\right)$ are equal to zero. 
Table 1. Main parameters of HOPG XANES spectrum at normal incidence extracted from Figure 2(a). The orientation parameter is $\mathrm{OP}=0.11$.

\begin{tabular}{|c|c|c|c|c|c|c|c|}
\hline Peaks & Literature & $\begin{array}{c}\text { Binding } \\
\text { energy }(e V)\end{array}$ & $\begin{array}{l}\text { Intensity } \\
\text { (c/s) }\end{array}$ & Assymetry & Base & Gaussian & $\begin{array}{l}\text { Final state band } \\
\text { and Brillouin zone }\end{array}$ \\
\hline A & Iijima 1991 & 285.46 & 0.43 & 0.10 & 0.14 & 0.60 & $\pi_{0}$ near Q \\
\hline A1 & $\begin{array}{l}\text { Eba } 2009 \\
\text { Yueh } 2001\end{array}$ & 287.21 & 0.21 & 0.00 & 0.00 & 1.20 & $\begin{array}{c}\text { Free-electron-like interlayer } \\
\text { state }+ \text { adsorption } \pi^{*} \mathrm{C}=\mathrm{C}-\mathrm{OH} \text { or } \\
-\left(\mathrm{C}=\mathrm{C}-\pi^{*} \mathrm{C}=\mathrm{O}\right)\end{array}$ \\
\hline A2 & This work & 288.49 & 0.34 & 0.00 & 0.00 & 2.60 & $\begin{array}{l}\text { Free-electron-like interlayer } \\
\text { state }+ \text { adsorption } \pi^{*} \mathrm{C}=\mathrm{C}\end{array}$ \\
\hline A3 & This work & 291.78 & 4.23 & 0.00 & 0.00 & 1.10 & $\sigma^{*} \mathrm{C}-\mathrm{O}$ \\
\hline $\mathbf{B}$ & Fayette 1998 & 292.93 & 3.80 & 0.07 & 0.16 & 0.90 & $\sigma_{1}, \sigma_{2}: \Gamma \rightarrow \mathrm{Q}$ \\
\hline
\end{tabular}

spectrum at normal incidence (normal orientation) is equal to 0.11 while the OP extracted from Table 2 of XANES spectrum at grazing incidence (parallel orientation) incidence is 0.69 .

We can observe that the $\pi^{*}$ resonance increased with the incidence angle from normal to grazing incidence angle. Reversely, the $\sigma^{*}$ resonance decreased with the incidence angle from normal to grazing incidence. This could be explained by the sine-square and cosine-square dependence of $\pi^{*}$ and $\sigma^{*}$ intensities, respectively as in Ref. [14]. It has been shown there that the intensity of $\pi^{*}$ transition is proportional to $\sin ^{2} \alpha$ (with its maximum at grazing incidence) and the intensity of $\sigma^{*}$ transition is proportional to $\cos ^{2} \alpha$ (with its maximum at normal incidence). The total extinction of A peak is supposed to obtain at normal incidence for good polarization of the synchrotron radiation X-ray beam, but, in our case, we do not observe this total extinction of $\pi^{*}$ band. This could be due to the incomplete polarization of X-ray beam light or sample misalignment [9].

\subsection{XANES Analysis of Orientation of CNTs Grown by DC HF CCVD Process}

Assuming that the intensities of the resonance are similar in graphite and carbon nanotubes, they can be estimated from a comparison to graphite XANES spectra. Typical CK-edge CNTs spectra, at normal $\left(\theta=0^{\circ}\right)$ and grazing $(\theta=\pi / 2)$ incidence angles are plotted and shown in Figures 3(a) and Figure 3(b), respectively. While observing carbon nanotubes XANES spectra, on the first point of view, they are very similar to graphite spectra.

In Figure 3(a) taken at normal incidence (normal orientation), specific peaks $A$ and $B$ are localized respectively at $285.34 \mathrm{eV}$ and $292.79 \mathrm{eV} . \mathrm{A}_{1}, \mathrm{~A}_{2}$ and $\mathrm{A}_{3}$ are localized at $287.34 \mathrm{eV}, 288.93 \mathrm{eV}$ and $289.51 \mathrm{eV}$, respectively. The peaks $A_{1}$ and $A_{2}$, regularly observed in the graphite XANES spectra [9] [10] [18] are also observed in our graphite and CNTs XANES spectra. As in graphite, $A_{1}$ is associated to the free-electron-like interlayer states in carbon nanotubes and corresponds to $\pi^{*}$ resonance. This feature could be due to the adsorption of some molecules such as $\mathrm{CO}, \mathrm{CO}_{2}$ and water [15]. $\mathrm{A}_{2}$, also attributed to the free-electron-like interlayer states, corresponds to $\pi^{*}$ resonance. It could be due to the adsorption of functionalized states $\mathrm{C}=\mathrm{C}$. $\mathrm{A}_{3}$ corresponds to $\sigma^{*} \mathrm{C}-\mathrm{O}$ bond resonance and is only observed in our CNTs spectra. We observed one more feature $\mathrm{A}_{4}$ at $291.65 \mathrm{eV}$ in our CNTs spectra. The peak $\mathrm{A}_{4}$ is currently observed on some HOPG and CNTs XANES spectra and corresponds to $\sigma^{*}$ exciton of $\mathrm{C}-\mathrm{H}^{*}$ bond resonance.

In Figure 3(b) taken at grazing incidence (parallel orientation), A and B are attributed to unoccupied $\pi^{*}$ resonance occurs at $285.62 \mathrm{eV}$ and to unoccupied $\sigma^{*}$ resonance occurs at $292.93 \mathrm{eV}$, respectively [10]. Their final-state band and Brillouin zone region are $\pi_{0}$ near $\mathrm{Q}$ at $2 \mathrm{eV}$ above the Fermi level and $\sigma_{1}, \sigma_{2}$ in the Brillouin region $\Gamma \rightarrow \mathrm{Q}$, respectively. Between the $\pi^{*}$ and $\sigma^{*}$ peaks, three one named $\mathrm{A}_{1}, \mathrm{~A}_{2}$ and $\mathrm{A}_{3}$ are found and localized at $287.48 \mathrm{eV}, 288.63 \mathrm{eV}$ and $289.92 \mathrm{eV}$, respectively. As at grazing incidence, $\mathrm{A}_{1}$ is associated to the freeelectron-like interlayer states in carbon nanotubes and corresponds to $\pi^{*}$ resonance. Mane et al. have indicated that this feature could be due to the adsorption of some molecules such as $\mathrm{CO}, \mathrm{CO}_{2}$ and water [15]. $\mathrm{A}_{2}$, also attributed to the free-electron-like interlayer states, corresponds to $\pi^{*}$ resonance. It could be due to the adsorption of functionalized states $\mathrm{C}=\mathrm{C}$. Regarding $\mathrm{A}_{3}$, it corresponds to $\sigma^{*} \mathrm{C}-\mathrm{O}$ bond resonance. $\mathrm{A}_{4}$ localized at $292.08 \mathrm{eV}$ corresponds to $\sigma^{*}$ exciton of $\mathrm{C}-\mathrm{H}^{*}$ bond resonance.

Figure 3(a) at normal incidence gives the intensities values $0.42 \mathrm{c} / \mathrm{s}$ of A peak of $\pi^{*}$ resonance and $2.63 \mathrm{c} / \mathrm{s}$ of B peak of $\sigma^{*}$ resonance. Figure 3(b) at grazing incidence gives the intensities values $2.39 \mathrm{c} / \mathrm{s}$ of A peak which corresponds to the $\pi^{*}$ resonance and $2.15 \mathrm{c} / \mathrm{s}$ of B peak of $\sigma^{*}$ resonance. 
Table 2. Main parameters of HOPG XANES spectrum at grazing incidence extracted from Figure 2(b). The orientation parameter is $\mathrm{OP}=0.69$.

\begin{tabular}{|c|c|c|c|c|c|c|c|}
\hline Peaks & Literature & $\begin{array}{c}\text { Binding } \\
\text { energy }(e V)\end{array}$ & Intensity (c/s) & Assymetry & Base & Gaussian & $\begin{array}{l}\text { Final state band and } \\
\text { Brillouin zone }\end{array}$ \\
\hline A & Iijima 1991 & 285.40 & 1.71 & 0.10 & 0.14 & 0.60 & $\pi_{0}$ near Q \\
\hline A1 & $\begin{array}{l}\text { Eba } 2009 \\
\text { Yueh } 2001\end{array}$ & 287.20 & 0.81 & 0.00 & 0.00 & 1.22 & $\begin{array}{c}\text { Free-electron-like interlayer } \\
\text { state }+ \text { adsorption } \\
\pi^{*} \mathrm{C}=\mathrm{C}-\mathrm{OH} \text { or } \\
-\left(\mathrm{C}=\mathrm{C}-\pi^{*} \mathrm{C}=\mathrm{O}\right)-\end{array}$ \\
\hline A2 & This work & 288.49 & 0.84 & 0.00 & 0.00 & 2.40 & $\begin{array}{l}\text { Free-electron-like interlayer } \\
\text { state }+ \text { adsorption } \pi^{*} \mathrm{C}=\mathrm{C}\end{array}$ \\
\hline A3 & This work & 291.78 & 2.48 & 0.00 & 0.00 & 1.10 & $\sigma^{*} \mathrm{C}-\mathrm{O}$ \\
\hline B & Fayette 1998 & 292.70 & 2.46 & 0.07 & 0.16 & 1 & $\sigma_{1}, \sigma_{2}: \Gamma \rightarrow \mathrm{Q}$ \\
\hline
\end{tabular}

As we observed for graphite spectra, we also observe the same trends in carbon nanotubes spectra showing that the $\pi^{*}$ resonance increased with the incidence angle from normal to grazing incidence angle. The $\sigma^{*}$ resonance has the reverse tendency in agreement with literature. The same explanation from graphite could be once more given for carbon nanotubes while they are very similar, thus, $\pi^{*}$ intensity strongly depends on the sinesquare while $\sigma^{*}$ intensity depends on cosine-square [14]. These authors demonstrated that the intensity of $\pi^{*}$ transition is proportional to $\sin ^{2} \alpha$ (with its maximum at grazing incidence) and the intensity of $\sigma^{*}$ transition is proportional to $\cos ^{2} \alpha$ (with its maximum at normal incidence). Contrary to graphite where the A peak is going to extinct at normal incidence for good polarization of the synchrotron radiation X-ray beam, this peak still remains high in carbon nanotubes spectra. This should be due to the carbon nanotubes defects. The OP parameter calculated from Table 3 and Table 4 is equal 0.16 at normal incidence angle and to 0.90 at grazing incidence angle, respectively. Those values prove the non total extinction of $\pi^{*}$ transition at grazing incidence when the $\pi$ orbitals are supposed to be lying parallel to the surface plane. Then, the non-extinction of the $\pi^{*}$ band may be attributed to the misalignment of CNTs relative to the surface normal, to the contributions steaming from other Carbon species or to the sample misalignment.

\subsection{Theoretical XANES Angular Dependence of HOPG and CNTs}

In this section, we focus on theoretical angular dependence of $\pi^{*}$ and $\sigma^{*}$ transitions. We used the values of $\pi^{*}$ and $\sigma^{*}$ intensities corresponding to peaks A and B, respectively, presented in section 3.1 and 3.2 for simulation with Matlab sofware. We assume a constant $\mathrm{N}$ value equal to 100 . We use different theoretical formula of XANES HOPG and CNTs at normal orientation (normal incidence) for two central issues of orientation light such as linear and circular polarization. We also use the formula corresponding to the parallel orientation (grazing incidence) for linear and circular polarization. The curves in all cases are plotted, shown and discussed in the following pages. By varying the incidence angle from normal to grazing incidence, the $\pi^{*}$ and $\sigma^{*}$ transitions could be more deepened as in Ref. [14] [17]. These authors have demonstrated that the $\pi^{*}$ and $\sigma^{*}$ intensities have sine-squared and cosine-squared dependencies, respectively. Showing that, with the increase incidence angle, the $\pi^{*}$ intensity was enhanced, while the $\sigma^{*}$ intensity was decreased. This has been explained by the fact that the CNTs are formed perpendicular to the substrate surface. In our work, we study the case of grazing and normal incidence by evaluating the angular dependence of HOPG and CNTs in linearly and circularly polarized light.

\subsubsection{Theoretical XANES Angular Dependence of HOPG}

We present in Figure 4 the superimposed HOPG $\pi^{*}$ and $\sigma^{*}$ state intensities versus the incidence angle in order to compare their angular dependence. The blue and green curves are $\pi^{*}$ intensities in linear and circular polarized light, respectively. The red and sky blue curves are $\sigma^{*}$ intensities in linear and circular polarized light, respectively. All are considered at normal orientation. The intensity is in the arbitrary units. When the $\pi^{*}$ intensity is in its minimum, the $\sigma^{*}$ intensity is in its maximum showing their variation in the reverse direction. We can observe that the $\pi^{*}$ intensities are too low in the linear and circular polarization comparing with $\sigma^{*}$. But, $\pi^{*}$ intensity in circular polarization is lower than the one in linear polarization. We also see that, $\sigma^{*}$ intensity in circular polarization is low while the one of linear polarization is high. The same behavior with the HOPG $\pi^{*}$ intensity is observed, however, the $\sigma^{*}$ intensity is observed in the negative part while the $\pi^{*}$ intensity is in the positive one. 


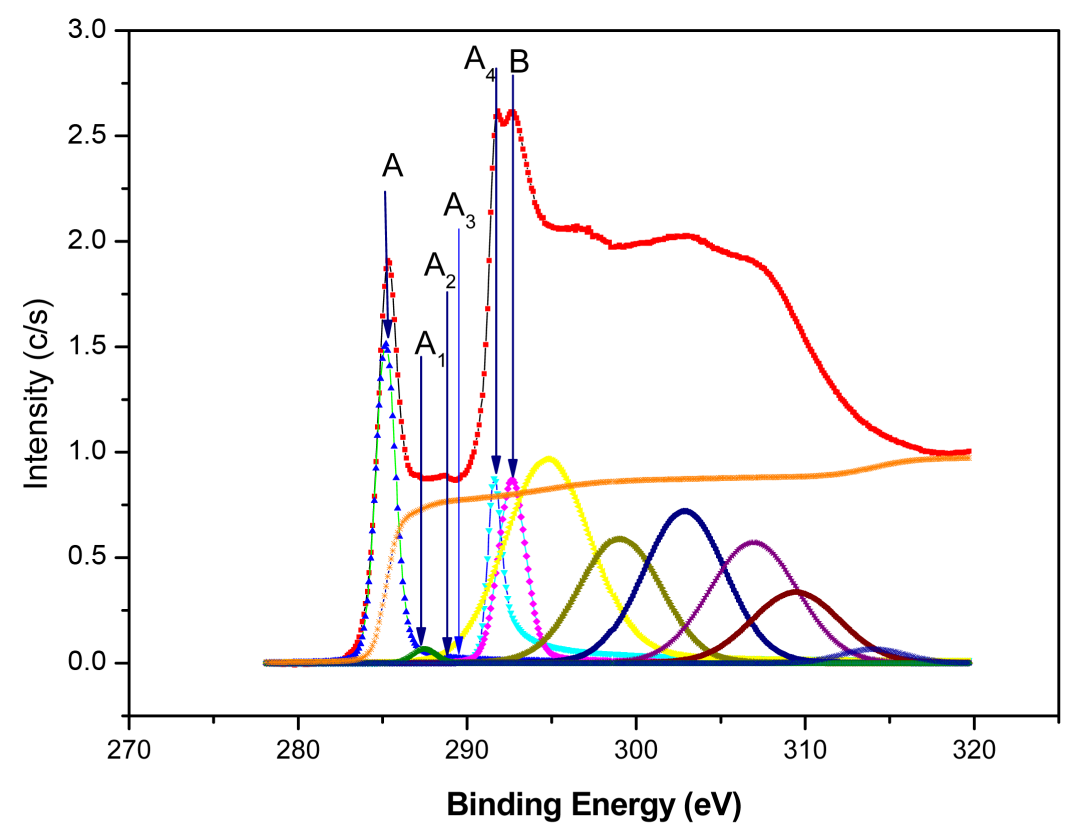

(a)

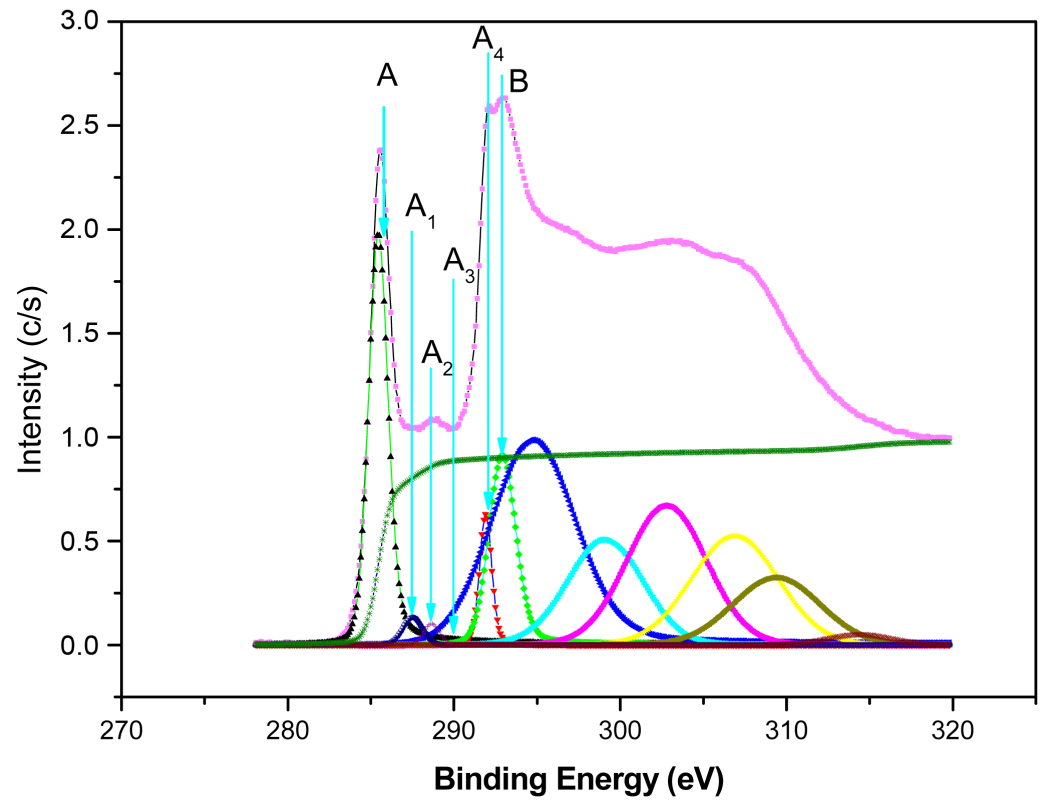

(b)

Figure 3. (a) Carbon nanotubes experimental and simulated superimposed XANES spectra at normal incidence. $A, A_{1}, A_{2}, A_{3}, A_{4}$ and $B$ are the features assigned to $\pi^{*}$ or $\sigma^{*}$ transitions. Their final state band and Brillouin zone are presented in Table 3. The asymmetry parameter of $A$ is equal to 0.10 while the one of $B$ is equal to 0.07 . The line base value of $\mathrm{A}$ is 0.15 while the one of $\mathrm{B}$ is 0.15 . The asymmetry and base line parameters of the intermediate peaks $\left(A_{1}, A_{2}, A_{3}\right.$ and $\left.A_{4}\right)$ are equal to zero. (b) Carbon nanotubes experimental and simulated superimposed XANES spectra at grazing incidence. $\mathrm{A}, \mathrm{A}_{1}, \mathrm{~A}_{2}, \mathrm{~A}_{3}, \mathrm{~A}_{4}$ and $\mathrm{B}$ are the features assigned to $\pi^{*}$ or $\sigma^{*}$ transitions. Their final state band and Brillouin zone are presented in Table 4 . The asymmetry parameter of $A$ is equal to 0.10 while the one of $B$ is equal to 0.07 . The line base value of $A$ and $B$ is 0.15 . The asymmetry and base line parameters of the intermediate peaks $\left(A_{1}, A_{2}, A_{3}\right.$ and $\left.A_{4}\right)$ are equal to zero. 


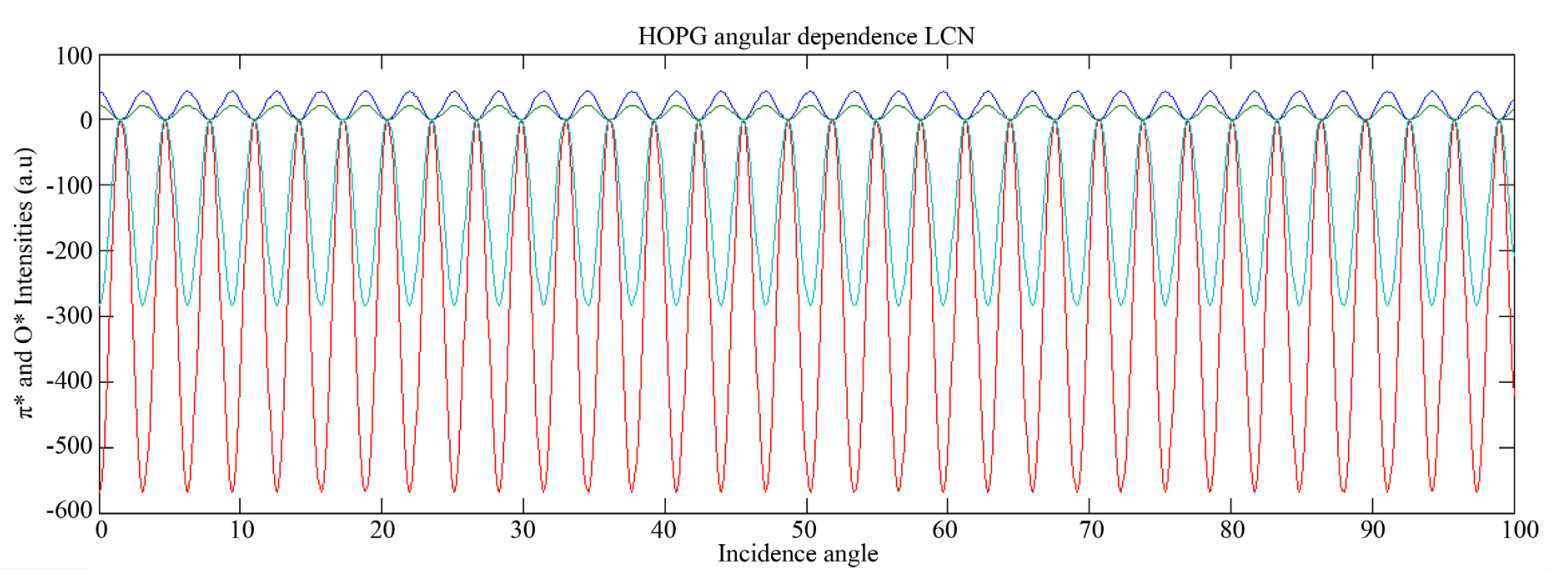

Figure 4. The comparison of HOPG $\pi^{*}$ and $\sigma^{*}$ state intensities versus incidence angle in linear and circular polarized light with the normal orientation (LCN). The blue and green curves are $\pi^{*}$ intensities in linear and circular polarization, respectively. The red and the sky blue curves are $\sigma^{*}$ intensities, respectively, in linear and circular polarization.

Table 3. Main parameters of CNTs XANES spectrum at normal incidence extracted from Figure 3(a). The orientation parameter is $\mathrm{OP}=0.16$.

\begin{tabular}{|c|c|c|c|c|c|c|c|}
\hline Peaks & Literature & $\begin{array}{c}\text { Binding } \\
\text { energy }(e V)\end{array}$ & $\begin{array}{c}\text { Intensity } \\
\text { (c/s) }\end{array}$ & Assymetry & Base & Gaussian & $\begin{array}{l}\text { Final state band and } \\
\text { Brillouin zone }\end{array}$ \\
\hline A & Iijima 1991 & 285.34 & 0.42 & 0.10 & 0.15 & 1 & $\pi_{0}$ near Q \\
\hline A1 & $\begin{array}{l}\text { Eba } 2009 \\
\text { Yueh } 2001\end{array}$ & 287.34 & 0.88 & 0.00 & 0.00 & 1.20 & $\begin{array}{l}\text { Free-electron-like } \\
\text { interlayer } \\
\text { state }+ \text { adsorption } \\
\pi^{*} \mathrm{C}=\mathrm{C}-\mathrm{OH} \text { or } \\
-\left(\mathrm{C}=\mathrm{C}-\pi^{*} \mathrm{C}=\mathrm{O}\right)-\end{array}$ \\
\hline A2 & This work & 288.93 & 0.9 & 0.00 & 0.00 & 1.72 & $\begin{array}{c}\text { Free-electron-like } \\
\text { interlayer state }+ \\
\text { adsorption } \pi^{*} \mathrm{C}=\mathrm{C}\end{array}$ \\
\hline A3 & This work & 289.51 & 0.89 & 0.00 & 0.00 & 0.60 & $\sigma^{*} \mathrm{C}-\mathrm{O}$ \\
\hline A4 & This work & 291.65 & 2.61 & 0.00 & 0.00 & 0.60 & $\sigma^{*}$ exciton \\
\hline B & Fayette 1998 & 292.79 & 2.63 & 0.07 & 0.15 & 1.25 & $\sigma_{1}, \sigma_{2}: \Gamma \rightarrow \mathrm{Q}$ \\
\hline
\end{tabular}

Table 4. Main parameters of CNTs XANES spectrum at grazing incidence extracted from Figure 3(b). The orientation parameter is $\mathrm{OP}=0.90$.

\begin{tabular}{|c|c|c|c|c|c|c|c|}
\hline Peaks & Literature & $\begin{array}{c}\text { Binding } \\
\text { energy }(e V)\end{array}$ & $\begin{array}{l}\text { Intensity } \\
\text { (c/s) }\end{array}$ & Assymetry & Base & Gaussian & $\begin{array}{l}\text { Final state band } \\
\text { and Brillouin zone }\end{array}$ \\
\hline $\mathbf{A}$ & Iijima 1991 & 285.62 & 2.39 & 0.10 & 0.15 & 1 & $\pi_{0}$ near $\mathrm{Q}$ \\
\hline A1 & $\begin{array}{l}\text { Eba } 2009 \\
\text { Yueh } 2001\end{array}$ & 287.48 & 1.05 & 0.00 & 0.00 & 1.15 & $\begin{array}{c}\text { Free-electron-like interlayer } \\
\text { state }+ \text { adsorption } \\
\pi^{*} \mathrm{C}=\mathrm{C}-\mathrm{OH} \text { or } \\
-\left(\mathrm{C}=\mathrm{C}-\pi^{*} \mathrm{C}=\mathrm{O}\right)-\end{array}$ \\
\hline A2 & This work & 288.63 & 1.08 & 0.00 & 0.00 & 2.20 & $\begin{array}{c}\text { Free-electron-like interlayer } \\
\text { state }+ \text { adsorption } \pi^{*} \mathrm{C}=\mathrm{C}\end{array}$ \\
\hline A3 & This work & 289.92 & 1.05 & 0.00 & 0.00 & 0.60 & $\sigma^{*} \mathrm{C}-\mathrm{O}$ \\
\hline A4 & This work & 291.88 & 2.59 & 0.00 & 0.00 & 0.60 & $\sigma^{*}$ exciton \\
\hline B & Fayette 1998 & 292.93 & 2.15 & 0.07 & 0.15 & 0.90 & $\sigma_{1}, \sigma_{2}: \Gamma \rightarrow \mathrm{Q}$ \\
\hline
\end{tabular}

In Figure 5, we also compare the HOPG $\pi^{*}$ and $\sigma^{*}$ state intensities in linear and circular polarized light with the parallel orientation. The $\pi^{*}$ corresponds to blue and green curves, while $\sigma^{*}$ corresponds to red and sky blue curves, respectively, in linear and circular polarization. We can observe low $\pi^{*}$ and $\sigma^{*}$ intensities in the circular 


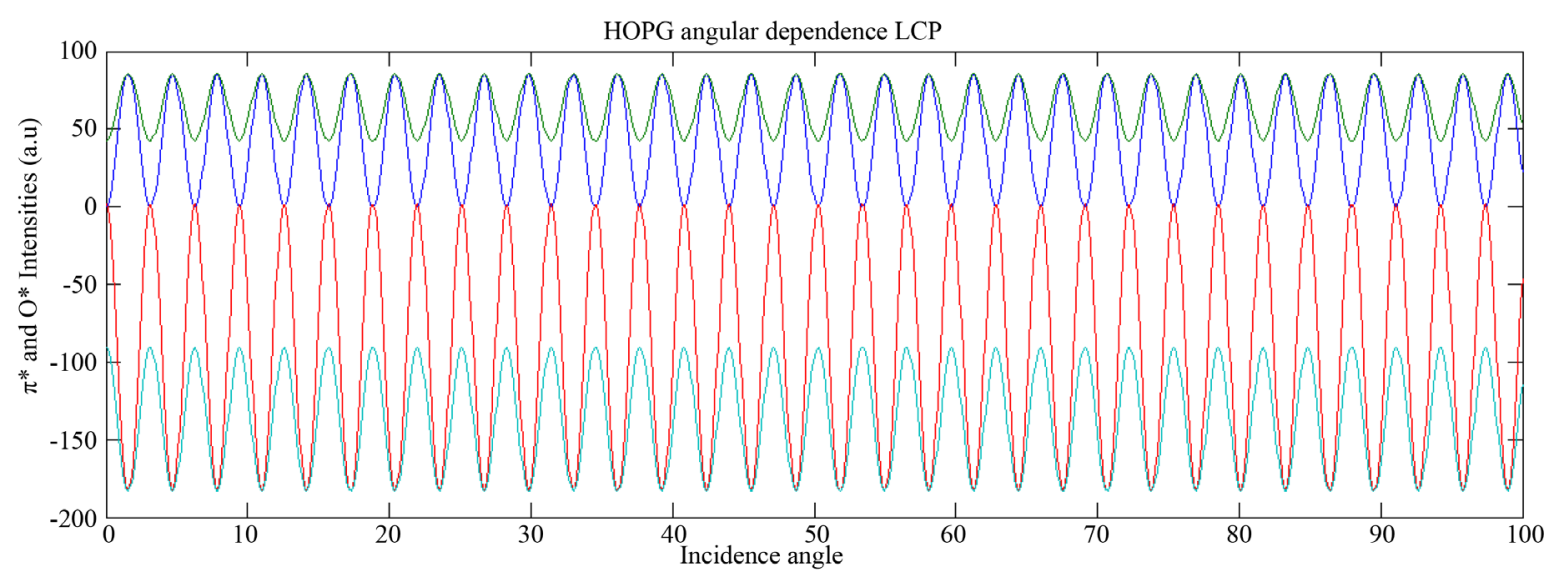

Figure 5. The comparison of HOPG $\pi^{*}$ and $\sigma^{*}$ state intensities versus incidence angle in linear and circular polarized light with the parallel orientation (LCP). The blue and green curves are $\pi^{*}$ intensities in linear and circular polarization, respectively. The red and sky blue curves are $\sigma^{*}$ intensities, respectively, in linear and circular polarization.

polarization while the high ones are observed in the linear polarization. We can observe that the $\sigma^{*}$ intensity in the circular polarization is lower than the one in the linear polarization. When the $\pi^{*}$ intensity is in its maximum, the $\sigma^{*}$ intensity is in its minimum showing their variation in the reverse direction. Obviously, this situation is opposite to the one of normal orientation. The $\pi^{*}$ intensities at parallel orientation are greater than those at normal orientation. This could be explain by the fact that for perfectly aligned HOPG or CNTs normally to the surface substrate, the $\pi^{*}$ transition should not be visible [10] at normal incidence since the $\pi^{*}$ orbitals are supposed to be lying parallel to the surface plane.

Figure 6 is the comparison of $\pi^{*}$ and $\sigma^{*}$ intensities versus square sine and square cosine, respectively, in the linear and circular polarization with normal and parallel orientation. The blue and green curves are in normal orientation while the red and sky blue are in parallel orientation, all, respectively in linear and circular polarized light.

At normal orientation, the $\pi^{*}$ intensities decrease and increase with square sine of incidence angle at parallel orientation. But the intensities in linear polarization decrease or increase very fast (Figure 6(a)). Observing Figure 6(b), the $\sigma^{*}$ intensities increase at normal orientation with the square cosine of incidence angle. But the intensity in linear polarization increases considerably. The reverse situation is observed at parallel orientation where the intensity decreases and the linear one strongly. The whole behavior of $\pi^{*}$ and $\sigma^{*}$ intensities are strongly depend on the incidence angle. Thus, we see on Figure 6 the sine square and cosine square angular dependence of $\pi^{*}$ and $\sigma^{*}$ intensities, respectively. However, for HOPG plots, the intensities at parallel orientation have not yet shown in the literature while those at normal orientation are in agreement with Zhongrui who has explained the sine square and cosine square dependencies of CNTs $\pi^{*}$ and $\sigma^{*}$ transitions [14]. This means that the experimental conditions should be very well care before, depending on the aim of the experiment.

\subsubsection{Theoretical XANES Angular Dependence of CNTs}

Figure 7 presents the comparison of $\pi^{*}$ and $\sigma^{*}$ intensities versus incidence angle in linear and circular polarized light, all with normal orientation. We can see that $\pi^{*}$ intensity in circular polarization is lower than $\pi^{*}$ intensity in linear polarization. The low $\sigma^{*}$ intensity in the circular polarization is observed while the high one is viewed in the linear polarization. $\pi^{*}$ intensity is in its minimum when $\sigma^{*}$ is in its maximum showing their reverse variation. $\pi^{*}$ intensity is observed in the positive region and $\sigma^{*}$ intensity is observed in the negative region.

Figure 8 is the comparison of $\pi^{*}$ and $\sigma^{*}$ intensities versus incidence angle in linear and circular polarized light, all with parallel orientation. In this figure, $\pi^{*}$ intensity in circular polarization is lower than $\pi^{*}$ intensity in linear polarization. The low $\sigma^{*}$ intensity in the circular polarization is observed while the high one is viewed in the linear polarization. $\pi^{*}$ intensity is observed in the positive region and $\sigma^{*}$ intensity is observed in the negative region. This behavior is perfectly similar to the one at normal orientation, nevertheless, on the one hand, $\pi^{*}$ intensity is in its maximum when $\sigma^{*}$ is in its minimum showing their reverse variation. On the other hand, $\pi^{*}$ intensity is smaller at normal orientation than the one at parallel orientation. As we concluded for HOPG, for per- 


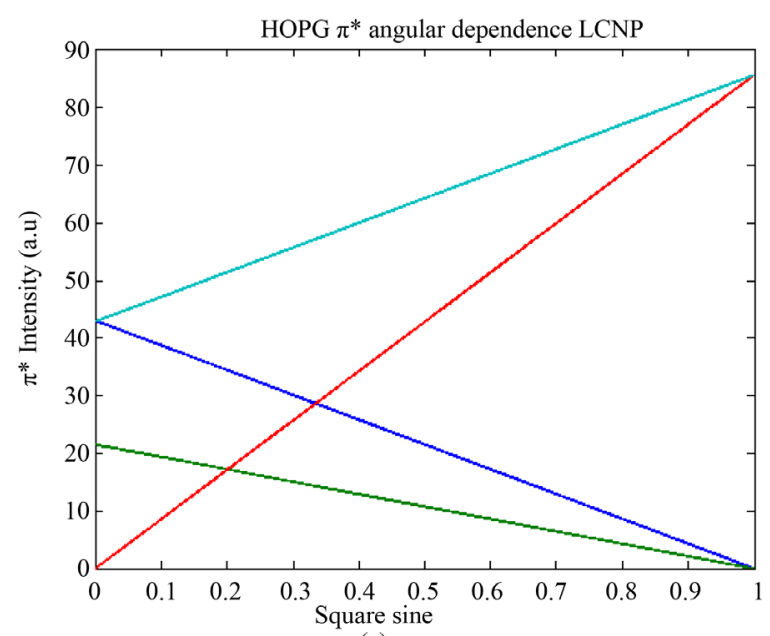

(a)

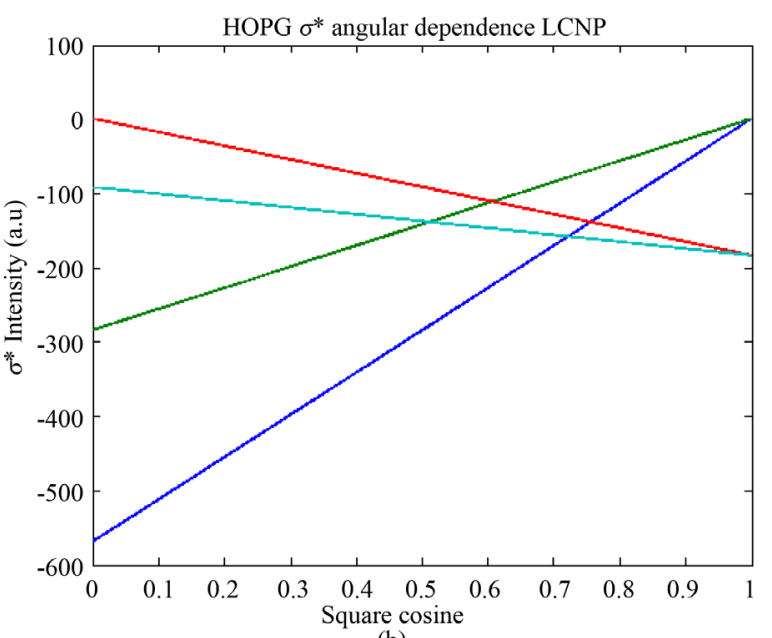

(b)

Figure 6. The comparison of HOPG (a) $\pi^{*}$ state intensities versus square sine of incidence angle; (b) $\sigma^{*}$ state intensities versus square cosine of incidence angle, both in linear and circular polarized light with the normal and parallel orientation. The blue lines are the $\pi^{*}$ and $\sigma^{*}$ intensities in linear polarization and the green lines are the $\pi^{*}$ and $\sigma^{*}$ intensities in circular polarization, both with normal orientation. The red lines and sky blue lines are the $\pi^{*}$ and $\sigma^{*}$ intensities, respectively, in linear and circular polarization, both with parallel orientation. LCNP denotes linear and circular polarization at normal and parallel orientation.

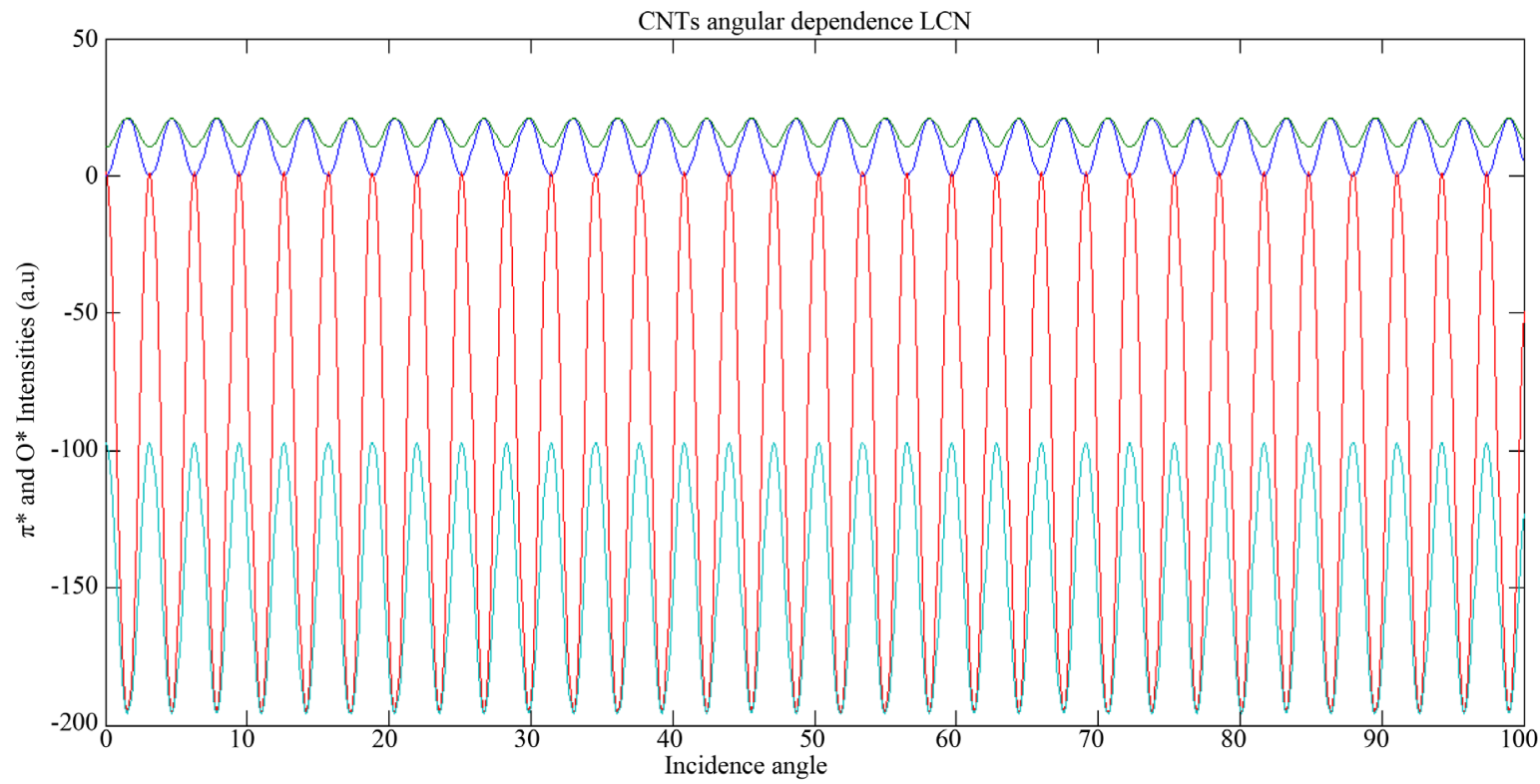

Figure 7. The comparison of CNTs $\pi^{*}$ and $\sigma^{*}$ state intensities in linear and circular polarized light with the normal orientation. The blue and green curves are the $\pi^{*}$ intensities, respectively, in linear and circular polarization. The red and sky blue ones are $\sigma^{*}$ intensities in linear and circular polarization, respectively.

fectly aligned HOPG or CNTs normally to the surface substrate, one should observe the extinction of $\pi^{*}$ transition [10] at normal incidence because in these conditions, the $\pi^{*}$ orbitals are supposed to be lying parallel to the surface plane. We noticed here the CNTs behavior similar to HOPG. But, we also see an important gap between the $\pi^{*}$ region and $\sigma^{*}$ region at parallel orientation. This allow us to conclude that, they is an existing region at parallel orientation where one does not expect any $\pi^{*}$ and $\sigma^{*}$ transitions because of the lack of polarization light.

Figure 9 is the plots of $\pi^{*}$ and $\sigma^{*}$ intensities versus square sine and square cosine of incidence angle, respectively, in linear and circular polarized light and with normal orientation 1) and parallel orientation 2). This figure 


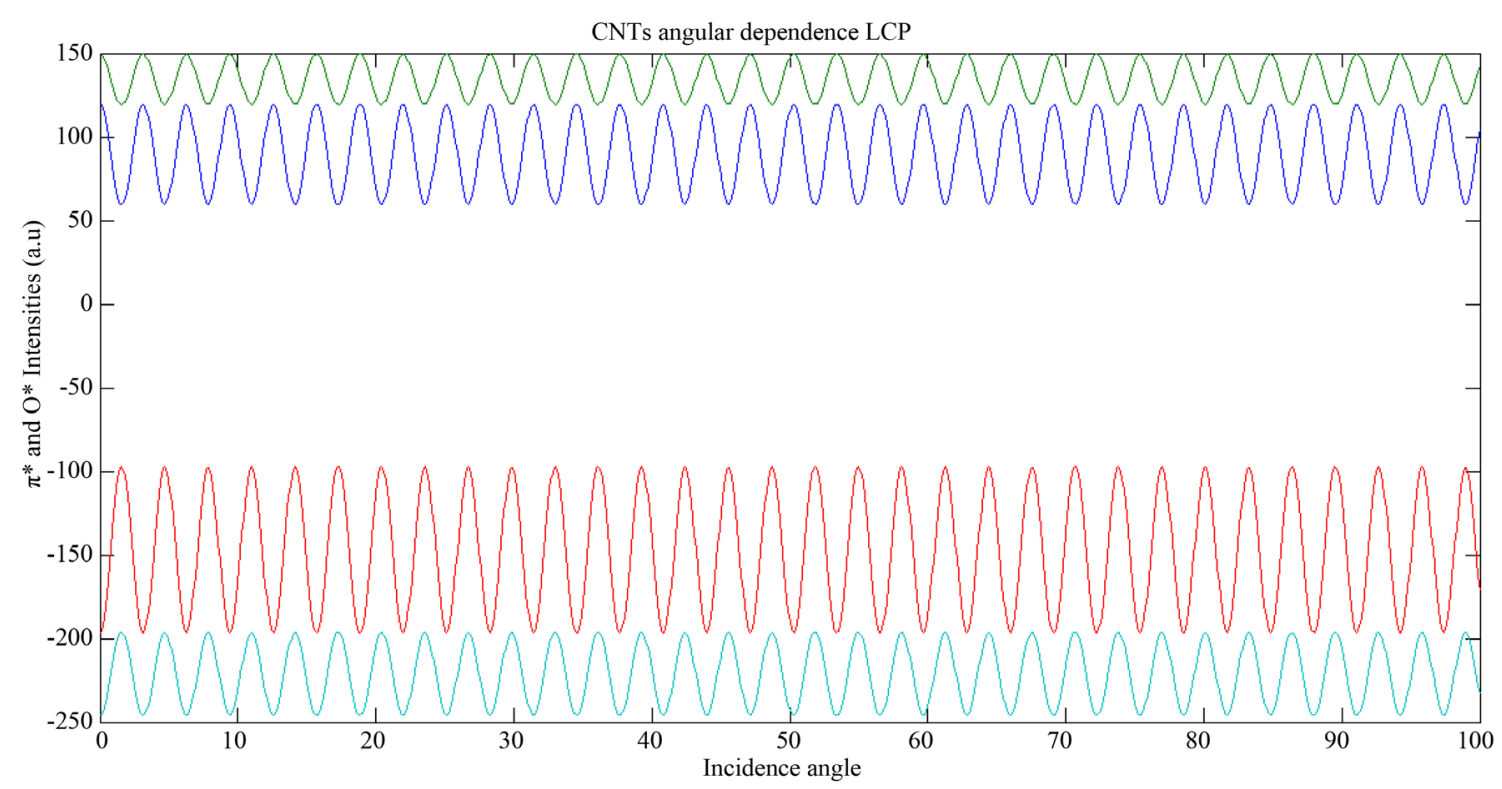

Figure 8. The comparison of CNTs $\pi^{*}$ and $\sigma^{*}$ state intensities in linear and circular polarized light with the parallel orientation. The blue and green curves are the $\pi^{*}$ intensities, respectively, in linear and circular polarization. The red and sky blue ones are $\sigma^{*}$ intensities in linear and circular polarization, respectively.

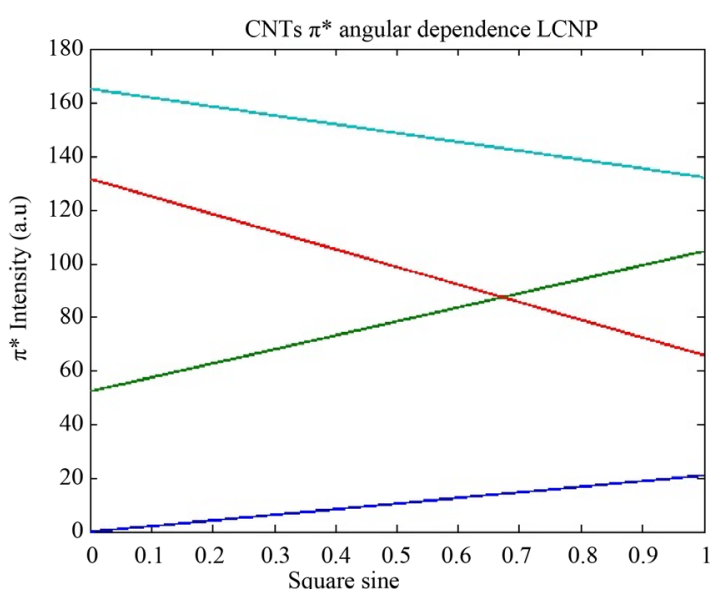

(a)

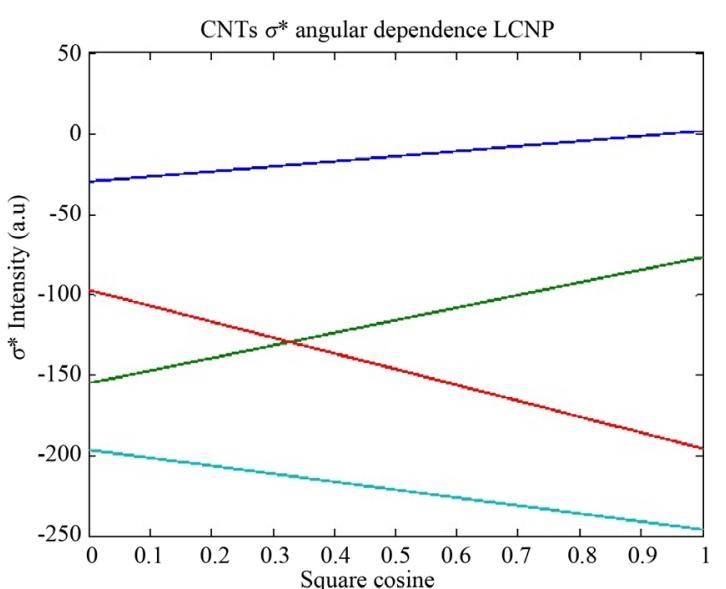

(b)

Figure 9. The comparison of CNTs $\pi^{*}$ state intensity versus square sine of incidence angle (a) and $\sigma^{*}$ state intensity versus square cosine of incidence angle (b) in linear and circular polarized light with the normal and parallel orientation. The blue lines are the $\pi^{*}$ and $\sigma^{*}$ intensities in linear polarization and the green lines are the ones in circular polarization, all of them with normal orientation. The red lines and sky blue lines are the $\pi^{*}$ and $\sigma^{*}$ intensities in linear and circular polarization, respectively, all of them with parallel orientation.

shows that, at normal orientation, $\pi^{*}$ intensity increases with square sine of incidence angle and $\sigma^{*}$ intensity increases with the square cosine of incidence angle. Whereas, at parallel orientation, $\pi^{*}$ and $\sigma^{*}$ intensities decrease with, respectively, square sine and square cosine of incidence angle. The general trend we noticed is the clearly sine square and cosine square dependencies of, respectively, $\pi^{*}$ and $\sigma^{*}$ intensities. The similarity between angular dependence of HOPG $\sigma^{*}$ and CNTs $\sigma^{*}$ is observed. However the variation of HOPG $\pi^{*}$ and CNTs $\pi^{*}$ is the reverse.

\section{Discussion}

Remarkably, all figures exhibiting the real dependence of angular dependence of $\pi^{*}$ and $\sigma^{*}$ intensities of HOPG 
and CNTs nanostructures. Nevertheless, the difference is observed between the $\pi^{*}$ and $\sigma^{*}$ intensities angular variation according to the literature. The sine-squared and cosine-squared dependencies of $\pi^{*}$ and $\sigma^{*}$ intensities, respectively, are well observed. Dependently on the position of the tube axis in $z$ direction (see Figure 2), it has been shown that the $\pi^{*}$ and $\sigma^{*}$ intensities of aligned carbon nanotube present respectively, sine-squared and cosine-squared dependencies [14]. This could be due to the presence of other isotropic carbon species (carbon clusters, diamond-like carbon or amorphous carbon) or to the randomly orientation of anisotropic nanotubes. It has been also shwon that the local contribution of $\sigma^{*}$ intensity is proportional to the sum of squared scalar products of two components and electric polarization vector [14], $\sigma_{\|}^{*}$ which is proportional to $2 \cos ^{2} \theta$ and $\sigma_{\perp}^{*}$ proportional to $\sin ^{2} \theta$. Therefore, the total $\sigma^{*}$ intensity decreases when the X-ray incidence angle changes from normal to grazing due to the cosine-square dependence.

Knowing that the polarization of the X-rays emerging from a storage ring depends on the line of sight, the polarization of the radiation is linear in that plane in the electrons' orbital plane (Figure 10(a)), so the electrons appear to oscillate in the horizontal plane. Thus, the $\pi^{*}$ orbitals are supposed to be lying parallel to the surface plane. The $\pi^{*}$ intensity could dominate at parallel orientation and its extinction although incomplete could be observed at normal orientation. This explains the high $\pi^{*}$ intensity observed at parallel orientation. Now, the position of the observer is important for the electrons' movement. On the one hand, if the observer is above the plane (Figure 10(b)) the electrons will appear and execute an elliptical orbit in a clockwise direction. The angular momentum from the observer's frame of reference is transferred to the emitted photons, which are left-circularly polarized (LeCP). The electric-field vector of the X-rays also rotates clockwise around the direction of propagation as viewed back down the beam from the observer. The angular momentum of the rotating electric field of the left-circular polarized is negative and is described by a vector of that magnitude pointing opposite to the direction of propagation. The photon will excite $\mathrm{L}_{1}$ level corresponding to C2pz states. In that case, the $\sigma^{*}$ resonance is a preference. This can explain the $\sigma^{*}$ intensities appearance in the negative region. In that conditions, the $\sigma^{*}$ intensity dominates at normal orientation and is minimized at parallel orientation.

On the other hand, if the observer is below the electrons' orbital plane (Figure 10(c)), the polarization is right-circular polarized and the photons carry a positive angular momentum. The photon will preferentially excite $\mathrm{L}_{3}$ levels which is $\mathrm{C} 2 \mathrm{p}_{\mathrm{y}}$ over the $\mathrm{L}_{2}$ corresponding C2 $\mathrm{p}_{\mathrm{x}}$ levels. In that case, the $\pi^{*}$ resonance is a preference and can explain its appearance in the positive region. The $\pi^{*}$ intensity dominates at parallel orientation as we noticed in LP, but a difference relative to the incidence angle exists and cannot be negligible. We can understand this particular trend to the study of experimental analysis based on $\pi^{*}$ transition. It could be better at parallel orientation instead of normal orientation, to use the beam light in linear polarization where $\beta=0^{\circ}$, to more evaluate the $\pi^{*}$ intensity transition. Meanwhile, the circular polarization is useful for the study of X-ray magnetic circular dichroism (XMCD).

\section{Conclusion}

We have investigated the XANES angular dependence of carbon nanotubes grown on plain substrates $\mathrm{SiO}_{2} /$

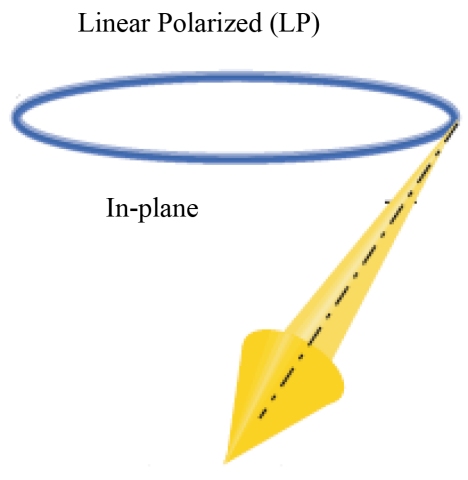

(a)

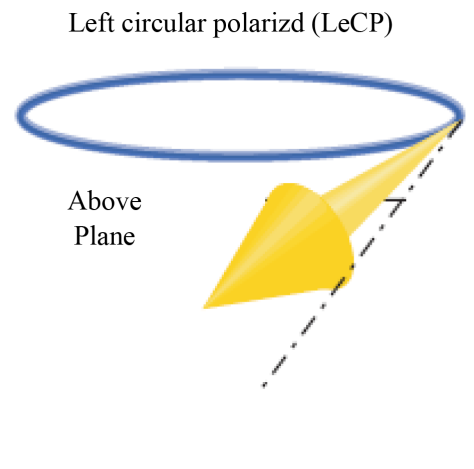

(b)

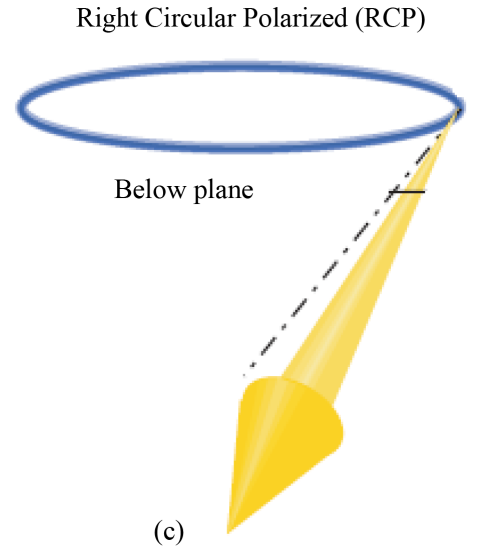

(c)

Figure 10. Different types of light polarization depending on the position of observer relative to plane of electron orbit. Here, LP denotes linear polarized, LeCP denotes Left-circular polarized while RCP is right circular polarized. 
Si(100) by a discharge current and hot filament-enhanced catalytic chemical vapor deposition process studied in the previous papers. For the good understanding of CNTs angular dependence, we have first analyzed XANES highly oriented pyrolitic graphite spectra and then XANES carbon nanotubes spectra at normal and grazing incidence. Main features were found and assigned to the $\pi^{*}$ and $\sigma^{*}$ resonances. The presence of free-interlayerlike electron was noticed around $\pi^{*}$ and $\sigma^{*}$ transitions. Experimental results of HOPG and CNTs show that the $\mathrm{C}-\mathrm{C} \pi^{*}$ resonance increases while the C-C $\sigma^{*}$ resonance decreases from normal to grazing incidence. This has been explained by the sine-square and cosine-square dependencies of $\pi^{*}$ and $\sigma^{*}$ intensities, respectively, in agreement with the literature. Theoretical XANES HOPG and CNTs curves exhibit the strong dependence on linearly or circularly polarized light of $\pi^{*}$ and $\sigma^{*}$ resonances. We noticed that, $\pi^{*}$ resonance was the priority one at the linear polarized light, as well as at right-circular polarized. The reverse tendency is observed at the leftcircular polarized light where $\sigma^{*}$ resonance is the priority one. The $\pi^{*}$ intensities are maximized at parallel orientation and the $\sigma^{*}$ intensities are minimized at normal orientation. This paper open new perspective on the dichroism study as X-ray magnetic linear dichroism (XMLD) used to deep the domain structure of anti ferromagnetic materials. So, as our carbon nanotubes are doped with metallic particles (Fe, Ni, Co), we could be able to investigate in the near future their ferromagnetic character.

\section{Acknowledgements}

P. Legagneux, Accosta, G. Schmerber and F Le Normand are acknowledged for providing experimental data. B. Thiodjio Sendja acknowledges E. Tchomgo Felenou for useful discussion and TRIL programme for financial support.

\section{References}

[1] Iijima, S. (1991) Helical Microtubules of Graphitic Carbon. Nature, 56, 354. http://dx.doi.org/10.1038/354056a0

[2] Melechko, A.V., Merkulov, V.I., McKnight, T.E., Guillon, M.A., Klein, K.L., Lowndes, D.H. and Simpson, M.L. (2005) Vertically Aligned Carbon Nanofibers and Related Structures: Controlled Synthesis and Directed Assembly. Journal of Applied Physics, 97, Article ID: 041301. http://dx.doi.org/10.1063/1.1857591

[3] Ren, Z.F., Huang, Z.P., Xu, J.W., Wang, J.H., Bush, P., Siegal, M.P. and Provencio, P.N. (1998) Synthesis of Large Arrays of Well-Aligned Carbon Nanotubes on Glass. Science, 282, 1105. http://dx.doi.org/10.1126/science.282.5391.1105

[4] Chhowalla, M., Teo, K.B.K., Ducati, C., Rupesinghe, N.L., Amaratunga, G.A.J., Ferrari, A.C., Roy, D., Robertson, J. and Milne, W.I. (2001) Growth Process Conditions of Vertically Aligned Carbon Nanotubes Using Plasma Enhanced Chemical Vapor Deposition. Journal of Applied Physics, 90, 5308. http://dx.doi.org/10.1063/1.1410322

[5] Cojocaru, C.S., Senger, A. and Le Normand, F. (2006) A Nucleation and Growth Model of Vertically-Oriented Carbon Nanofibers or Nanotubes by Plasma-Enhanced Catalytic Chemical Vapor Deposition. Journal of Nanoscience and Nanotechnology, 6, 1331. http://dx.doi.org/10.1166/jnn.2006.144

[6] Mane, J., Cojocaru, C.S., Barbier, A., Deville, J.P., Thiodjio Sendja, B. and Le Normand, F. (2007) GISAXS Study of the Alignment of Oriented Carbon Nanotubes Grown on Plain SiO2/Si(100) Substrates by a Catalytically Enhanced CVD Process. Physica Status Solidi (a), 1, 21.

[7] Thiodjio Sendja, B., Eba Medjo, B., Mane Mane, J., Ben Bolie, G., Diop, D. and Owono, A. (2010) A GISAXS Study of the Angular Dependence of Carbon Nanotubes Grown on a Plain Substrate by the DC HF CCVD Process. Physica Scripta, 82, Article ID: 025601. http://dx.doi.org/10.1088/0031-8949/82/02/025601

[8] Rosenberg, R.A., Love, P.J. and Rehn, V. (1986) Polarisation-Dependant Near-Edge X-Ray-Absorption Fine Structure of Graphite. Physical Review B, 33, 4034. http://dx.doi.org/10.1103/PhysRevB.33.4034

[9] Fischer, D.A., Wentzcovitch, R.M., Carr, P.G., Continenza, A. and Freeman, A.J. (1991) Graphitic Interlayer States-A Carbon-K Nearedge X-Ray-Absorption Fine-Structure Study. Physical Review B, 44, 1427. http://dx.doi.org/10.1103/PhysRevB.44.1427

[10] Eba Medjo, R., Thiodjio Sendja, B., Mane Mane, J. and Owono Ateba, P. (2009) XAS Study of the Orientation of Oriented Carbon Nanotube Films. Physica Scripta, 80, Article ID: 055602. http://dx.doi.org/10.1088/0031-8949/80/05/055602

[11] Chiou, J.W., Yueh, C.L., Jan, J.C., Tsai, H.M., Pong, W.F., Hong, I.-H., Klauser, R., Tsai, M.-H. and Chang, Y.K. (2002) Electronic Structure of the Carbon Nanotube Tips Studied by X-Ray-Absorption Spectroscopy and Scanning Photoelectron Microscopy. Applied Physics Letters, 81, 4189. http://dx.doi.org/10.1063/1.1523152

[12] Tang, Y.H., Sham, T.K., Hu, Y.F., Lee, C.S. and Lee, S.T. (2002) Near-Edge X-Ray Absorption Fine Structure Study 
of Helicity and Defects in Carbon Nanotubes. Chemical Physics Letters, 366, 636. http://dx.doi.org/10.1016/S0009-2614(02)01620-2

[13] Schiessling, J., Kjeldgaard, L., Rohmund, F., Falk, L.K.L., Campbell, E.E.B., Nordgren, J. and Bruhwiler, P.A. (2003) Synchrotron Radiation Study of the Electronic Structure of Multiwalled Carbon Nanotubes. Journal of Physics: Condensed Matter, 15, 6563. http://dx.doi.org/10.1088/0953-8984/15/38/022

[14] Li, Z.R., Zhang, L., Resasco, D.E., Mun, B.S. and Requejo, F.G. (2007) Angle-Resolved X-Ray Absorption near Edge Structure Study of Vertically Aligned Single-Walled Carbon Nanotubes. Applied Physics Letters, 90, Article ID: 103115. http://dx.doi.org/10.1063/1.2709506

[15] Mane, J.M., Le Normand, F., Medjo, R.E., Cojocaru, C.S., Ersen, O., Senger, A., Laffon, C., Sendja, B.T., Biouele, C.M., Ben-Bolie, G.H., Ateba, P.O. and Parent, P. (2014) Alignment of Vertically Grown Carbon Nanostructures Studied by X-Ray Absorption Spectroscopy. Materials Sciences and Applications, 5, 966-983. http://dx.doi.org/10.4236/msa.2014.513098

[16] Stöhr (1992) Analysis of K-Shell Excitation Spectra by Curve Fitting. Journal of NEXAFS Spectroscopy, 25, 211-238. http://dx.doi.org/10.1007/978-3-662-02853-7_7

[17] Hemraj-Benny, T., Banerjee, S., Sambasivan, S., Balasubramanian, M., Fischer, D.A., Eres, G., Puretzky, A.A., Geohegan, D.B., Lowndes, D.H., Han, W.Q., Misewich, J.A. and Wong, S.S. (2005) Near-Edge X-Ray Absorption Fine Structure Spectroscopy as a Tool for Investigating Nanomaterials. Small, 2, 26-35. http://dx.doi.org/10.1002/smll.200500256

[18] Pate, B.B., Hecht, M.H., Binns, C., Lindau, I. and Spicer, W.E. (1982) Photoemission and Photon-Stimulated Ion Desorption Studies of Diamond(111): Hydrogen. Journal of Vacuum Science \& Technology, 21, 364-367. http://dx.doi.org/10.1116/1.571781 